Editorial

\title{
Fractional Calculus: Theory and Applications
}

\author{
Francesco Mainardi
}

Department of Physics and Astronomy, University of Bologna, and The National Institute of Nuclear Physics (INFN), Via Irnerio, 46, I-40126 Bologna, Italy; francesco.mainardi@bo.infn.it; Tel.: +39-051-209-1068

Received: 22 July 2018; Accepted: 17 August 2018; Published: 21 August 2018

Fractional calculus is allowing integrals and derivatives of any positive order (the term fractional is kept only for historical reasons). It can be considered a branch of mathematical physics that deals with integro-differential equations, where integrals are of convolution type and exhibit mainly singular kernels of power law or logarithm type.

It is a subject that has gained considerably popularity and importance in the past few decades in diverse fields of science and engineering. Efficient analytical and numerical methods have been developed but still need particular attention.

The purpose of this Special Issue is to establish a collection of articles that reflect the latest mathematical and conceptual developments in the field of fractional calculus and explore the scope for applications in applied sciences.

The papers in this Special Issue can be divided according to the following scheme considering their main purposes:

(1) Analytical Theory

(2) Numerical Methods

(3) Applications

\section{Analytical Theory}

We start with a brief note by the Guest Editor Francesco Mainardi [1]: A Note on the Equivalence of Fractional Relaxation Equations to Differential Equations with Varying Coefficients. This equivalence is indeed shown for the simple fractional relaxation equation for which the solution in terms of the Mittag-Leffler function is known. This simple argument may lead to the equivalence of more general processes governed by evolution equations of fractional order with constant coefficients to processes governed by differential equations of integer order but with varying coefficients. Our main motivation is to solicit researchers to extend this approach to other areas of applied science to have a deeper knowledge of certain phenomena, both deterministic and stochastic ones, investigated nowadays with the techniques of fractional calculus.

Then, we consider two notes about the fractional Marchaud derivative from different perspectives that surely constitute a novelty in the actual literature of fractional calculus.

In the paper by Fausto Ferrari [2]: Weyl and Marchaud Derivatives: A Forgotten History, the author recalls the contribution given by Hermann Weyl and André Marchaud to the notion of fractional derivative. In addition, he discusses some relationships between the fractional Laplace operator and Marchaud derivative in the perspective to generalize these objects to different fields of the mathematics.

The aim of the paper by Sergei Rogosin and Maryna Dubatovskaya [3]: Letnikov vs. Marchaud: A Survey on Two Proinent Constructions of Fractional Derivatives, is to present the essence of two important approaches in Fractional Calculus, namely, those developed by Letnikov (or by Grünwald and Letnikov) and by Marchaud. The authors collect here the most important results for the corresponding fractional derivatives, compare these constructions and highlight their role in Fractional Calculus and its applications. 
In the paper by Trifce Sandev [4]: Generalized Langevin Equation and the Prabhakar Derivative, the generalized Langevin equation is considered with regularized Prabhakar derivative operator. The author analyzes the mean square displacement, time-dependent diffusion coefficient and velocity autocorrelation function. Further, he introduces the so-called tempered regularized Prabhakar derivative and analyzes the corresponding generalized Langevin equation with friction term represented through the tempered derivative.

In the paper by Roberto Garra, Enzo Orsingher and Federico Polito [5]: A Note on Hadamard Fractional Differential Equations with Varying Coefficients and Their Applications in Probability, the authors establish a connection between some generalizations of the COM-Poisson distributions and integro-differential equations with time-varying coefficients involving Hadamard integrals or derivatives. Moreover, they suggest a new interesting application in probability of a recently introduced generalized Le Roy function (see [6]).

In the paper by Yuri Luchko [7]: On Some New Properties of the Fundamental Solution to the Multi-Dimensional Space- and Time-Fractional Diffusion-Wave Equation, the Mellin-Barnes integrals technique is employed to deduce some new analytical properties of solutions to the multi-dimensional space- and time-fractional diffusion-wave equation. Indeed, some new closed-form formulas for particular cases of the fundamental solution are derived. In particular, the author solves the open problem of the representation of the fundamental solution to the two-dimensional neutral-fractional diffusion-wave equation in terms of the known special functions.

In the paper by Khadidja and Lamine Nisse [8]: An Iterative Method for Solving a Class of Fractional Functional Differential Equations with "Maxima", the authors deal with nonlinear fractional differential equations with "maxima" and deviating arguments. The nonlinear part of the problem under consideration depends on the maximum values of the unknown function taken in time-dependent intervals. Proceeding by an iterative approach, they obtain the existence and uniqueness of the solution, in a context that does not fit within the framework of fixed-point theory methods for the self-mappings, frequently used in the study of such problems. An example illustrating their main result is also given.

\section{Numerical Methods}

The paper "Numerical Solution of Fractional Differential Equations: A Survey and a Software Tutorial" by Roberto Garrappa [9] aims to provide a tutorial for the numerical solution of fractional differential equations (FDEs). In particular, numerical methods for solving systems of FDEs, as well as of multi-order type (i.e., in which each equation has a different order), and multi-term FDEs (i.e., equations in which derivatives of different order appears in the same equation), are presented. Some aspects related to the efficient implementation of the methods are discussed and the corresponding MATLAB routines are made freely available.

The paper "Numerical Solution of Multiterm Fractional Differential Equations Using the Matrix Mittag-Leffler Functions" by Marina Popolizio [10] focuses on a numerical approach to solve Multiterm Fractional Differential Equations (MTFDEs), that is, equations involving derivatives of different orders. They are very common to model many important processes, particularly for multi-rate systems. The analyzed approach is based on the possibility to equivalently write MTFDEs in terms of a linear system of Fractional Differential Equations of the same order; the, $\mathrm{n}$ the solution is computed by means of the Mittag-Leffler function evaluated in the coefficient matrix by means of very recent tools [11]. This matrix approach turns out to be very accurate and fast, also in comparison with other numerical methods, as shown by several numerical tests presented in the paper.

The paper by Vladimir D. Zakharchenko and Ilya G. Kovalenko [12]: Best Approximation of the Fractional Semi-Derivative Operator by Exponential Series, considers the implementation of a fractional-differentiating filter of the order of $1 / 2$ by a set of automation astatic transfer elements, which greatly simplifies practical implementation. Real technical devices have the ultimate time delay, albeit small in comparison with the duration of the signal. As a result, the real filter will process 
the signal with some error. In accordance with this, this paper introduces and uses the concept of a "pre-derivative" of $1 / 2$ of magnitude. An optimal algorithm for realizing the structure of the filter is proposed based on the criterion of minimum mean square error. Relations are obtained for the quadrature coefficients that determine the structure of the filter. This technique is shown to be useful for a significant reduction in the time required to obtain an estimate of the mean frequency of the spectrum of Doppler signals when seeking to measure the instantaneous velocity of dangerous near-Earth cosmic objects.

\section{Applications}

Among the many interesting applications of fractional calculus to physical systems, in this Special Issue, we find the paper devoted to the fractional viscoelasticity.

In the paper "Storage and Dissipation of Energy in Prabhakar Viscoelasticity" by Ivano Colombaro, Andrea Giusti and Silvia Vitali [13], the authors clarify some aspects of the attenuation processes emerging in a Fractional Maxwell model of viscoelasticity involving Prabhakar derivatives. On this topic we refer the reader to A. Giusti and I. Colombaro [14].

A further application related to fractional calculus is devoted to the free electron laser (FEL) and carried out by a group led by a well-known specialist on this topic (Prof. Dattoli). In the paper "Fractional Derivatives, Memory Kernels and Solution of a Free Electron Laser Volterra Type Equation" by Marcello Artioli, Giuseppe Dattoli, Silvia Licciardi and Simonetta Pagnutti [15], the authors recall that the high gain FEL equation is a Volterra type integro-differential equation amenable for analytical solutions in a limited number of cases. In this note, a novel technique, based on an expansion employing a family of two variable Hermite polynomials, is shown to provide straightforward analytical solutions for cases hardly solvable with conventional means. The possibility of extending the method by the use of expansion using different polynomials (such as two variable Legendre) expansion is also discussed.

The paper "Application of Tempered-Stable Time Fractional-Derivative Model to Upscale Subdiffusion for Pollutant Transport in Field-Scale Discrete Fracture Networks" by Bingqing Lu, Yong Zhang, Donald M. Reeves, HongGuang Sun and Chunmiao Zheng [16] aims to explore the relationship between real-world aquifer properties and non-Fickian transport dynamics. According to the authors, the fractional partial differential equations built upon fractional calculus can be reliably applied with appropriate hydro-geologic interpretations. They use the Monte Carlo approach to generate field-scale multiple discrete fracture network (DFN) flow and transport scenarios where the fracture properties change systematically, and then to simulate groundwater flow and pollutant transport through the complex DFNs. For a point source located initially in the mobile phase or fracture, the late-time behavior for the pollutant breakthrough curves (BTCs) simulated by the Monte Carlo approach is then explained by the tempered-stable time fractional advection-dispersion equation. The relationship between medium heterogeneity and transport dynamics through the combination of numerical experiments and stochastic analysis is built.

In the paper by Guoxing Lin [17]: Analysis of PFG Anomalous Diffusion via Real-Space and Phase-Space Approaches, two significantly different methods are proposed to analyze the pulsed-field gradient (PFG) anomalous diffusion: the effective phase-shift diffusion equation (EPSDE) method and a method based on observing the signal intensity at the origin. The EPSDE method describes the phase evolution in virtual phase space, while the method to observe the signal intensity at the origin describes the magnetization evolution in real space. However, these two approaches give the same general PFG signal attenuation including the finite gradient pulse width (FGPW) effect, which can be numerically evaluated by a direct integration method. The direct integration method is fast and without overflow. It is a convenient numerical evaluation method for Mittag-Leffler function-type PFG signal attenuation. The methods here provide a clear view of spin evolution under a field gradient, and their results will help the analysis of PFG anomalous diffusion.

Funding: This research received no external funding. 
Conflicts of Interest: The author declares no conflict of interest.

\section{References}

1. Mainardi, F. A Note on the Equivalence of Fractional Relaxation Equations to Differential Equations with Varying Coefficients. Mathematics 2018, 6, 8. [CrossRef]

2. Ferrari, F. Weyl and Marchaud Derivatives: A Forgotten History. Mathematics 2018, 6, 6. [CrossRef]

3. Rogosin, S.; Dubatovskaya, M. Letnikov vs. Marchaud: A Survey on Two Prominent Constructions of Fractional Derivatives. Mathematics 2018, 6, 3. [CrossRef]

4. Sandev, T. Generalized Langevin Equation and the Prabhakar Derivative. Mathematics 2017, 5, 66. [CrossRef]

5. Garra, R.; Orsingher, E.; Polito, F. A Note on Hadamard Fractional Differential Equations with Varying Coefficients and Their Applications in Probability. Mathematics 2018, 6, 4. [CrossRef]

6. Garrappa, R.; Rogosin, S.; Mainardi, F. On a Generalized Three-Parameter Wright Function of Le Roy Type. Fract. Calc. Appl. Anal. 2017, 20, 1196-1215. [CrossRef]

7. Luchko, Y. On Some New Properties of the Fundamental Solution to the Multi-Dimensional Space- and Time-Fractional Diffusion-Wave Equation. Mathematics 2017, 5, 76. [CrossRef]

8. Nisse, K.; Nisse, L. An Iterative Method for Solving a Class of Fractional Functional Differential Equations with "Maxima". Mathematics 2017, 6, 2. [CrossRef]

9. Garrappa, R. Numerical Solution of Fractional Differential Equations: A Survey and a Software Tutorial. Mathematics 2018, 6, 16. [CrossRef]

10. Popolizio, M. Numerical Solution of Multiterm Fractional Differential Equations Using the Matrix Mittag-Leffler Functions. Mathematics 2018, 6, 7. [CrossRef]

11. Garrappa, R.; Popolizio, M. Computing the Matrix Mittag-Leffler Function with Applications to Fractional Calculus. J. Sci. Comput. 2018. [CrossRef]

12. Zakharchenko, V.; Kovalenko, I. Best Approximation of the Fractional Semi-Derivative Operator by Exponential Series. Mathematics 2018, 6, 12. [CrossRef]

13. Colombaro, I.; Giusti, A.; Vitali, S. Storage and Dissipation of Energy in Prabhakar Viscoelasticity. Mathematics 2018, 6, 15. [CrossRef]

14. Giusti, A.; Colombaro, I. Prabhakar-like fractional viscoelasticity. Commun. Nonlinear Sci. Numer. Simul. 2018, 56, 138-143. [CrossRef]

15. Artioli, M.; Dattoli, G.; Licciardi, S.; Pagnutti, S. Fractional Derivatives, Memory Kernels and Solution of a Free Electron Laser Volterra Type Equation. Mathematics 2017, 5, 73. [CrossRef]

16. Lu, B.; Zhang, Y.; Reeves, D.; Sun, H.; Zheng, C. Application of Tempered-Stable Time Fractional-Derivative Model to Upscale Subdiffusion for Pollutant Transport in Field-Scale Discrete Fracture Networks. Mathematics 2018, 6, 5. [CrossRef]

17. Lin, G. Analysis of PFG Anomalous Diffusion via Real-Space and Phase-Space Approaches. Mathematics 2018, 6, 17. [CrossRef]

(c) 2018 by the author. Licensee MDPI, Basel, Switzerland. This article is an open access article distributed under the terms and conditions of the Creative Commons Attribution (CC BY) license (http:// creativecommons.org/licenses/by/4.0/). 